\title{
Changing Characters' Point of View in Interactive Storytelling
}

\author{
Fred Charles \\ School of Computing \\ Teesside University \\ Middlesbrough, U.K. \\ f.charles@tees.ac.uk
}

\author{
Julie Porteous \\ School of Computing \\ Teesside University \\ Middlesbrough, U.K. \\ j.porteous@tees.ac.uk
}

\author{
Marc Cavazza \\ School of Computing \\ Teesside University \\ Middlesbrough, U.K. \\ m.o.cavazza@tees.ac.uk
}

\begin{abstract}
Virtual characters are at the epicentre of Interactive Storytelling systems and in recent years multiple AI planning approaches have been described to specify their autonomous behaviour. This demonstrator provides an overview of our novel approach to the definition of virtual characters aimed at achieving a balance between character autonomy and global plot structure which proposes the notion of a character's Point of View. Additionally, the demonstrator offers the active spectator the ability to discover the story described from the perspective of a number of different characters. We present our fully-implemented Interactive Narrative based on Shakespeare's Merchant of Venice. The system, which features a novel AI planning approach to story generation, can generate very different stories depending on the Point of View adopted and support dynamic modification of the story world which results in different story consequences.
\end{abstract}

\section{Categories and Subject Descriptors}

H.5.1 [Multimedia Information Systems]: Artificial, augmented and virtual realities -Virtual Reality for Art and Entertainment

\section{General Terms}

Algorithms, Design, Experimentation, Theory.

\section{Keywords}

Interactive Storytelling, Narrative Modelling, Planning, Virtual Reality, Virtual Actors.

\section{PRINCIPLES}

Our novel approach to the definition of characters' behaviours is based on the concept of Point of View (PoV) which describes one character's perspective on the story. Our work was originally initiated by an attempt at revisiting the duality between character and plot at a representational level. We observed that in earlier work in character-based storytelling [1], key narrative actions had to be either distributed over individual characters' actions, or described as the actions of a principal character. Our working hypothesis is that a given narrative action (such as a contract, a betrayal, a challenge, and so on ...) can be represented differently depending on the perspective of each character taking part in that action. In other words, a PoV consists of a character's representation defined from the perspective of the overall plot, not just of the character's role independent of any other. The PoV also implements the naive concept of a given character's standpoint on a set of events, although in an a priori rather than a posteriori fashion. This is achieved by defining different representations for the same narrative action depending on the PoV, which in turn requires, for instance, different sets of pre (resp. post) conditions. With such representations, narrative generation will adopt a given character's PoV for the selection of the actual narrative action, thus resulting in story variants according to the PoV. In addition, these variants will respond differently to real-time modifications of the narrative domains such as those introduced by user interaction.

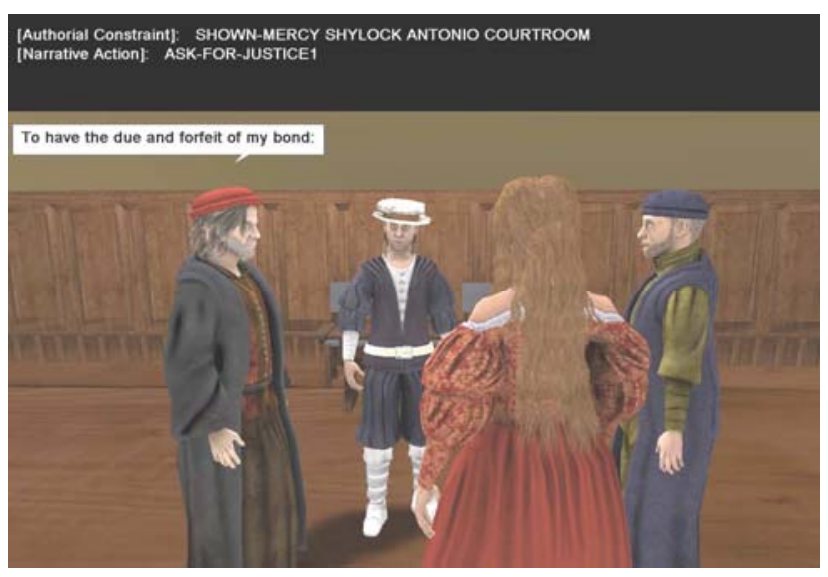

Figure 1.3D virtual stage and characters from the Merchant of Venice Interactive Storytelling system: Shylock, Bassanio, Portia and Antonio. 
To illustrate the concept, we have developed an Interactive Narrative [4] based on Shakespeare's Merchant of Venice ${ }^{1}$ [6], which is notorious for offering multiple interpretations depending on which character's perspective is considered. However, rather than offering post hoc interpretations, our system is actually able to generate different stories depending on the PoV adopted. Here, we investigate the capacities of this new approach [4] to allow the user to dynamically modify the story world through the selection of a specific PoV, and experience how it will result in different consequences for the unfolding of the story.

[4] provides a more complete description of the novel approach which is based on authorial control over the story definition using constraints [3]. It also shows how a baseline plot can be adapted in order to be told from the perspective of one of the feature characters. This method ensures that the essence of the plot is retained, rather than simply being projected onto a character's role. The real impact of this approach could be to reconcile narrative generation with modern scriptwriting which often takes characters as its starting point. An ability to explore various realisations of the narrative according to the characters' perspective would be a powerful tool to explore the narrative space. In the objective of extending our approach to real-time Interactive Storytelling, we have created this demonstrator as a new benchmark to further experiment the potential for dynamic modifications of story worlds by users.

\section{SYSTEM ARCHITECTURE}

In line with the traditional architecture of an Interactive Storytelling system [2], our prototype (Figure 2) comprises: i) a visualisation engine featuring virtual characters on a $3 \mathrm{D}$ stage (Figure 1), based on the UT2003 ${ }^{\mathrm{TM}}$ game engine; ii) a plan-based narrative engine generating characters' high-level actions; iii) a single input module, which uses a Nintendo Wiimote controller as input device. Our narrative generation engine features a novel decomposition approach with a control mechanism that dynamically selects constraints that are used to structure the narrative trajectory [4]. The different characters' PoV are represented declaratively using one invariant for each character which can take one of two values representing the opposing PoV's for that character.

The user is visualising on a $2 \mathrm{D}$ screen the dramatisation of the sequence of narrative actions generated by the plan-based narrative engine. The Interactive Storytelling system ensures the user has the possibility to interact with the unfolding plot at anytime, whereby she may select a different PoV from which the story world is to be presented.

\section{INTERACTIONS}

For example, in the Merchant of Venice, Shylock's PoV can be that he is a victim or that he is ruthless which could be represented as: (pov shylock-victim) or (pov shylock-ruthless). Antonio's PoV can be that he is a victim or a carefree risk taker, represented as: (pov antoniovictim) or (pov antonio-risk-taker). The PoV is set

\footnotetext{
${ }^{1}$ Our inspiration for this work is based on the modern interpretation depicted in the 2004 filmic adaptation by Michael Radford [5].
}

initially and remains unchanged for the duration of a particular narrative until the user decides to switch to the PoV of another character. Figure 3 presents an example narrative which illustrates how a narrative can switch between PoV's within the same generated narrative, thus simulating changes introduced by user interaction. We can notice that the initial segment of this narrative (on the left-hand side) the PoV is (pov antonio-risktaker). Another variation in narrative content can be observed in the narrative for PoV (pov shylock-victim), which is presented on the right-hand-side of the figure 3

The final segment of the narrative shown in figure 3 returns to a $\mathrm{PoV}$ of (pov antonio-risk-taker). Here, variation is possible since the final narrative action chosen enables Antonio to follow a slightly different line of conduct and show gratitude to the court for freeing him.

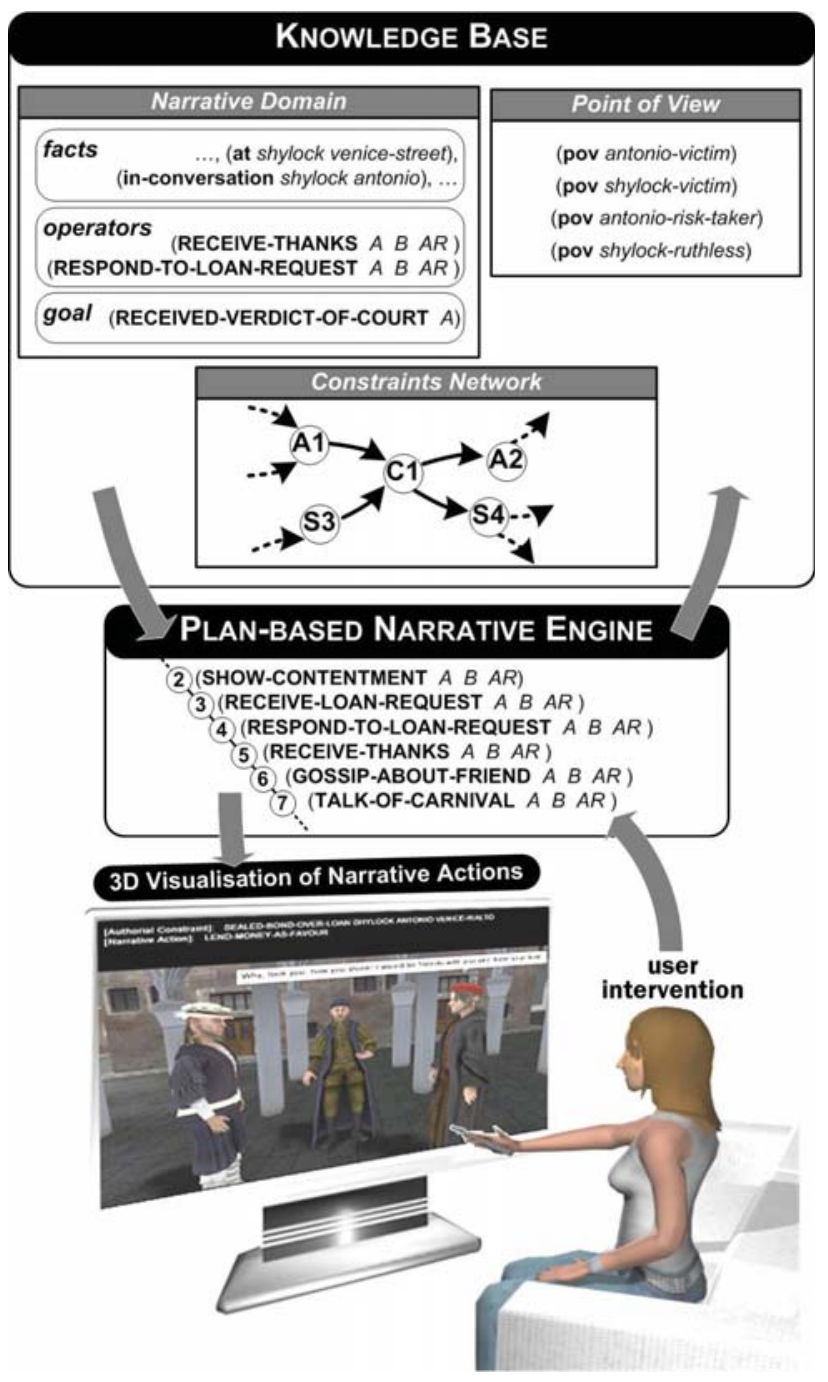

Figure 2. Overall system architecture. 


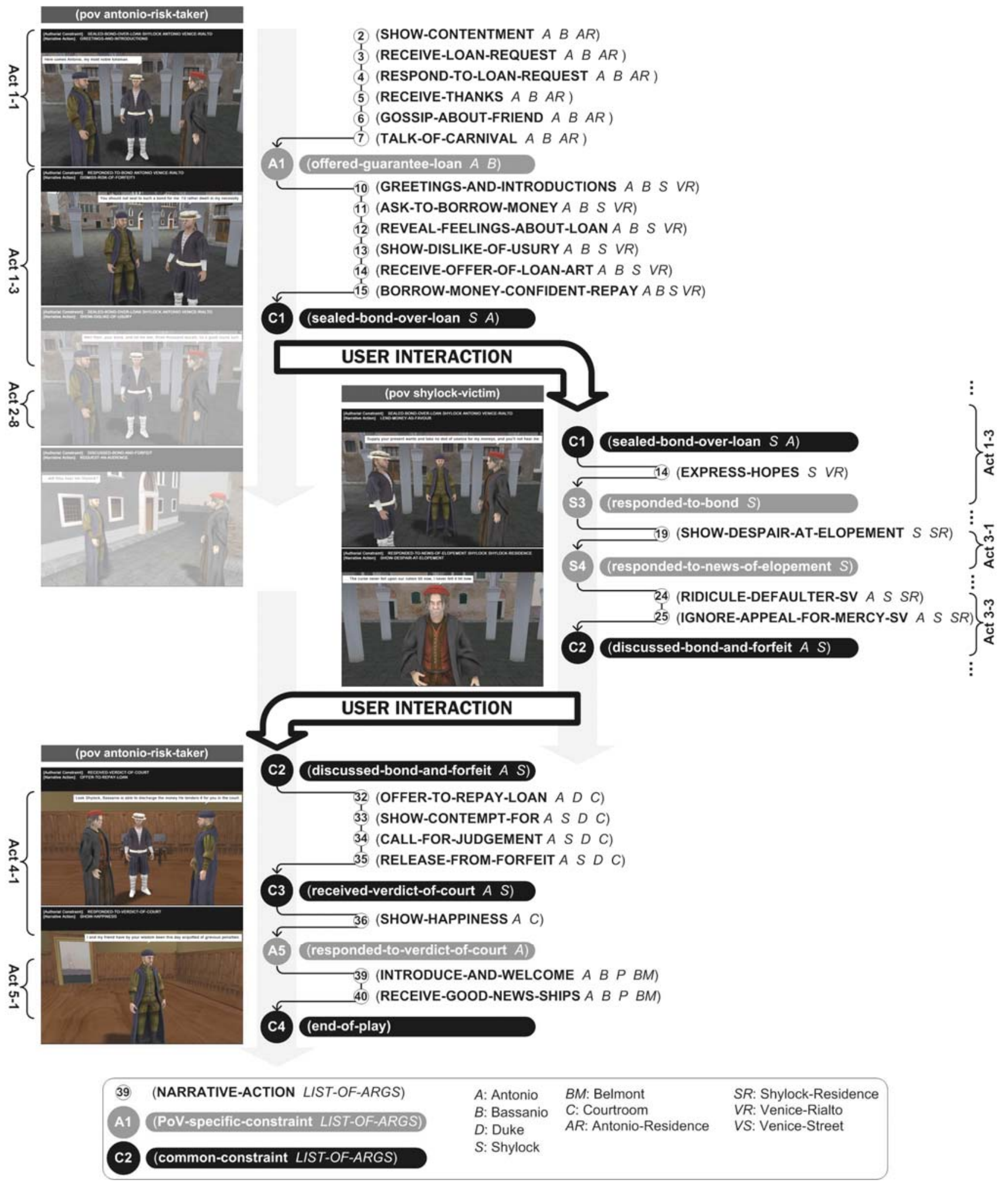

Figure 2. Example of a sequence of narrative actions generated by the plan-based narrative engine, for two different PoVs (Antonio-risk-taker and shylock-victim), as well as the potential influence on the narrative generated when user interaction occurs. 


\section{CONCLUSION}

This demonstrator is both meant to provide evidence of the many narrative variations which can be generated by the narrative engine, based on the principles of characters' point of view, and also demonstrate the capabilities of the approach while reincorporating user interaction into an interactive storytelling framework.

We have shown how a baseline plot can be adapted in order to be told from the perspective of one of the feature characters. The real impact of this approach could be to reconcile narrative generation with modern scriptwriting which often takes characters as its starting point. One of the key benefits of this new approach is also an ability to explore various instantiations of the narrative according to the characters' perspective. As the story world can be explored several times in repetition, there is a real potential for the creation of a tool which would support the exploration of the whole narrative space.

\section{ACKNOWLEDGMENTS}

This work has been funded (in part) by the European Commission under grant agreement IRIS (FP7-ICT-231824).

\section{REFERENCES}

[1] Cavazza, M., Charles, F., and Mead, S. J. 2002. CharacterBased Interactive Storytelling. IEEE Intelligent Systems 17, 4 (July 2002), 17-24.

[2] Cavazza, M., Pizzi, D., Charles, F., Vogt, T., and André, E. 2009. Emotional input for character-based interactive storytelling. In Proceedings of the 8th international Conference on Autonomous Agents and Multiagent Systems - Volume 1 (Budapest, Hungary, May 10 - 15, 2009), 313320.

[3] Porteous, J. and Cavazza, M. 2009. Controlling Narrative Generation with Planning Trajectories: The Role of Constraints. In Proceedings of the 2nd Joint international Conference on interactive Digital Storytelling: interactive Storytelling (Guimarães, Portugal, December 09 - 11, 2009). I. A. Iurgel, N. Zagalo, and P. Petta, Eds. Lecture Notes In Computer Science, vol. 5915. Springer-Verlag, Berlin, Heidelberg, 234-245.

[4] Porteous, J., Cavazza, M. and Charles, F. 2010. Narrative Generation through Characters' Point of View. In Proceedings of the 9th International Conference on Autonomous Agents and Multiagent Systems (Toronto, Canada, May 2010).

[5] Radford, M. 2004. MGM Home Ent. (Europe) Ltd., The Merchant of Venice (film adaptation), 2004.

[6] Shakespeare, W. 2007. The Merchant of Venice. Penguin Popular Classics (New Edition), 2007. 